\title{
DETERMINANTES DEL GASTO DE LOS HOGARES EN SALUd EN MÉXICO
}

\author{
Hada M. Sáenz-Vela y Ángela M. Guzmán-Giraldoª
}

Fecha de recepción: 28 de mayo de 2020. Fecha de aceptación: 3 de noviembre de 2020.

https://doi.org/10.22201/iiec.20078951e.2021.205.69644

Resumen. El gasto de bolsillo y catastrófico en México persiste a lo largo de la distribución del ingreso, y la afiliación a determinadas instituciones de salud no exime a los individuos de enfrentar estas situaciones. Se estiman modelos tobit para analizar los determinantes de los gastos de bolsillo y catastróficos en salud, encontrando que las características regionales, individuales y del hogar provocan heterogeneidad en el comportamiento del gasto en salud. De acuerdo con los datos, el uso efectivo de los servicios públicos reduce, aunque no suficientemente, las probabilidades de incurrir en gastos excesivos. Las políticas públicas deben de encaminarse a la promoción de la salud y prevención de enfermedades, así como a reducir las brechas en la calidad de los servicios públicos de salud.

Palabras clave: gasto en salud; distribución del ingreso; servicios públicos; prevención de enfermedades; modelos tobit.

Clasificación JEL: C51; I14; I15; I18; N36; P46.

\section{DETERMINANTS OF HOUSEHOLD HEALTH SPENDING IN MEXICO}

\begin{abstract}
In Mexico, out-of-pocket and catastrophic spending are persistent across the income distribution spectrum, and affiliation with health institutions does not safeguard individuals against such possibilities. This study estimates tobit models to analyze the determinants of out-of-pocket and catastrophic health expenditures, and finds that regional, individual, and household characteristics cause heterogeneity in health spending behaviors. The data suggest that the effective use of public services reduces, albeit insufficiently, the probability of incurring excessive expenditures. Public policies should focus on health promotion and disease prevention, as well as on reducing the gaps in the quality of public health services.
\end{abstract}

Key Words: health expenditure; income distribution; public services; disease prevention; tobit models.

\footnotetext{
${ }^{a}$ Universidad Autónoma de Coahuila, México. Correos electrónicos: hada.saenz@uadec.edu.mx y angelamelissa08@gmail.com, respectivamente.
} 


\section{INTRODUCCIÓN}

De acuerdo con diversos autores, el gasto de bolsillo en salud por parte de los hogares sirve como un indicador de la desigualdad en el sistema de salud (Xu et al., 2007; Van Minh et al., 2013; Cinaroglu, 2018). Esto es representativo especialmente en países de ingresos bajos y medio-bajos (Mills, 2014), aunque los países desarrollados no están exentos (oms, 2017; Baird, 2016). Incluso, el Objetivo 3 de los Objetivos de Desarrollo Sostenible (oDs), en su numeral 8 señala que la cobertura universal incluye la protección contra riesgos financieros, para lo cual considera como indicador la proporción de hogares con grandes gastos en salud como porcentaje del gasto del hogar (onU, 2019). El gasto en salud se vuelve catastrófico cuando un hogar debe reducir su consumo básico por un periodo para hacer frente a los gastos en salud en un porcentaje mayor a un determinado umbral (Xu et al., 2003). Por lo tanto, comprender los factores que pueden tener una mayor incidencia en la presencia de gastos excesivos en salud permite ofrecer propuestas de políticas públicas mejor enfocadas a la reducción de las brechas entre la población.

El sistema de salud en México se rige bajo un modelo sanitario mixto en el que intervienen tanto actores públicos como privados. Aunado a ello, Martínez y Murayama (2016) señalan que en el caso mexicano el derecho a la salud se encuentra diferenciado de acuerdo con la institución de afiliación, pues la protección es mayor cuando se obtiene como una prestación laboral, y se da en un entorno donde una gran parte de la población se desempeña en ocupaciones informales. ${ }^{1}$ De esta forma, contar con seguridad social (afiliación como prestación laboral) reduce la probabilidad de presentar gastos catastróficos (Knaul et al., 2018; Granados-Martínez y Nava-Bolaños, 2019), aunque ésta parece incrementarse cuando los individuos no asalariados están afiliados a programas asociados a servicios de la Secretaría de Salud ${ }^{2}$ (Díaz-González y Ramírez-García, 2017). A ello se añade que la población que no cuenta con ninguna de estas formas de afiliación, o que encuentra alguna barrera para su

1 De acuerdo con Instituto Nacional de Estadística y Geografía (INEGI, 2018), la Tasa de Informalidad Laboral 1 (TILI), reportada por el INEGI para el tercer trimestre de 2018, alcanzó 56.7\%, aproximadamente 30.6 millones de personas. De dicho conjunto, 14.8 millones contó con actividad en el sector informal (Tasa de Ocupación en el Sector Informal 1) que representó 27.5\% de la población ocupada; y 15.8 millones se encontraron en condiciones de informalidad (el vínculo laboral no es reconocido por su fuente de trabajo).

2 Como señalan Gómez-Dantés et al. (2011), el sector de trabajadores no asalariados suele recurrir a servicios de la Secretaría de Salud, de las secretarías estatales de Salud y el IMss-Oportunidades (llamado después IMss-Prospera), a lo que se sumó el programa del Seguro Popular a partir de enero de 2004 . 
uso, puede incrementar sustancialmente la probabilidad de demandar servicios privados (Bautista-Arredondo et al., 2014).

El presente texto se plantea desde el objetivo de identificar las variables que ayudan a explicar las diferencias entre los niveles de gasto de bolsillo y el gasto catastrófico en salud en México. Para ello, se emplearán características del individuo y del hogar, así como el efecto del gasto público per cápita, lo que potencialmente puede generar divergencias regionales. La comparación entre el gasto de bolsillo y el catastrófico, así como el alcance del gasto público resultan en una contribución a la literatura reciente sobre el tema del gasto en salud en el país. De esta forma, el documento se compone de seis secciones, incuida la introducción, inicia con una revisión sobre los antecedentes del sistema de salud en México, y continúa con la literatura enfocada en el análisis de los gastos excesivos en salud en el país. La cuarta sección corresponde a la metodología a utilizar, para seguir con el análisis de los resultados econométricos, para cerrar con algunas reflexiones e implicaciones de política.

\section{EL SISTEMA DE SALUD EN MÉXICO}

Siguiendo a Gómez-Dantés et al. (2011) y a Martínez y Murayama (2016), el sistema mexicano de salud se compone de un sector privado y uno público. El sector privado ofrece servicio sólo a aquellos individuos con capacidad de pago a proveedores y aseguradores privados; no obstante, el sector público es más complejo. Por un lado, está el subsector público del Sistema de Seguridad Social que atiende a trabajadores formales y a sus familias, así como a jubilados; dentro de este rubro se encuentra el Instituto Mexicano del Seguro Social (IMSs), el Instituto de Seguridad y Servicios Sociales de los Trabajadores del Estado (Issste, tanto federal como estatal), y los servicios que se ofrecen a trabajadores de Petróleos Mexicanos (pemex), Secretaría de la Defensa Nacional (SEDENA) y Secretaría de Marina (SEMAR). El otro subsector público es el Sistema de Protección Social en Salud (sPss), dirigido a aquellos individuos que no son derechohabientes de la seguridad social, donde la Secretaría de Salud (ssa) coordina también a los Servicios Estatales de Salud (sESA), el IMSsOportunidades (llamado después IMss-Prospera, IMss-P) y el Seguro Popular (sp).

La Encuesta Nacional de Ingreso y Gasto de los Hogares (ENIGH) de 2018 reporta que cerca del $86 \%$ de la población estaba afiliada a algún servicio de salud. En la tabla 1, según datos reportados por el Consejo Nacional de Evaluación de la Política de Desarrollo Social (CONEvaL), se muestra la evolución 
Hada M. Sáenz-Vela y Ángela M. Guzmán-Giraldo

Tabla 1. Porcentaje de población afiliada según institución

\begin{tabular}{lrrrrrr}
\hline $\begin{array}{l}\text { Afiliación según } \\
\text { institución }\end{array}$ & 2008 & 2010 & 2012 & 2014 & 2016 & 2018 \\
\hline Seguro Popular (SP) & 19.3 & 30.5 & 40.8 & 43.5 & 45.3 & 42.2 \\
IMSS & 31.2 & 31.8 & 32.1 & 34.0 & 35.4 & 36.4 \\
ISSSTE-ISSSTE estatal & 6.6 & 7.3 & 6.6 & 6.8 & 7.0 & 6.9 \\
PEMEX-SEDENA-SEMAR & 0.9 & 0.9 & 0.9 & 1.0 & 0.8 & 0.8 \\
IMSS-Prospera & - & - & - & - & 1.0 & 0.3 \\
Otros & 2.7 & 2.3 & 2.6 & 2.1 & 1.6 & 1.5 \\
\hline
\end{tabular}

Fuente: adaptado de CONEVAL (2019).

de la distribución de la población afiliada entre los diversos esquemas de salud pública, aunque una persona pudiera estar inscrita en más de una institución, situación que se ha ido corrigiendo (ssA, 2019a). La población asegurada en las entidades asociadas a los esquemas de empleo formal se mantuvo relativamente constante en el periodo 2008-2018, mientras que el sp presentó los mayores incrementos en el mismo periodo. Esto se debe al objetivo de cobertura universal de los servicios de salud implementado en México (García-Junco, 2012).

El brazo operativo del sPss, el sp surgió como un programa presupuestal que empezó a funcionar a partir de 2004. El objetivo primordial del sP era lograr la cobertura de servicios de salud a través de la afiliación pública y voluntaria para individuos carentes de seguridad social, así como protección financiera a dicho grupo poblacional (Martínez y Murayama, 2016). Siguiendo a Flamand y Moreno-Jaimes (2015), una innovación considerable de este esquema fue que los recursos se distribuían según el número de afiliados, al tiempo que se aprovechaba la infraestructura existente y capacidad operativa de las entidades federativas, lo cual, señalan los autores, implicó a su vez diferencias en efectividad y calidad de los servicios de salud. Así, el sp utilizaba infraestructura y recursos de la SSA y los SESA y, eventualmente, del IMSS y proveedores privados (Gómez-Dantés et al., 2011).

El gasto público total en salud que se destina al financiamiento del sistema de salud se conforma a partir de distintos fondos. De acuerdo con la Dirección General de Información en Salud (ssa, 2019b), el gasto público se divide entre la Secretaría de Salud (Ramo 12), el Imss-Prospera (Ramo 19), el Fondo de Aportaciones para los Servicios de Salud (fASSA-Ramo 33), el Gasto 
Estatal, la sedena (Ramo 7), la semar (Ramo 13), el Imss, el Issste, pemeX, el Instituto de Seguridad Social para las Fuerzas Armadas Mexicanas (ISSFAM) y las Instituciones de Seguridad Social de las Entidades Federativas (ISSES). La información respecto de estos niveles de gasto se encuentra disponible en el Sistema de Cuentas en Salud a Nivel Federal y Estatal (sicuentas, 2019).

El financiamiento del sistema de salud en México presenta desequilibrios importantes, según la condición de aseguramiento y entre entidades federativas. Murillo y Almonte (2020) indican que, entre 2014 y 2018, 53.8\% del gasto público se destinó a instituciones de seguridad social, mientras que $46.2 \%$ se asignó a programas para población sin derechohabiencia; aunque la reducción de la brecha entre estas proporciones se dio en gran medida después de la introducción del sp, como lo apuntan Martínez y Murayama (2016). Sin embargo, estos autores señalan que la diversidad de criterios demográficos, económicos y administrativos propiciaron segmentación y heterogeneidad de los servicios, debido a que las fuertes divergencias entre entidades federativas pueden explicarse por la importancia del empleo formal en los mercados laborales estatales y por los rezagos en la cobertura de salud.

La SSA (2019a) también ofrece algunas observaciones importantes, destacando que el gasto público ha tenido un comportamiento diferenciado por estados, ligeramente decreciente en el periodo 2012-2017; y el gasto en servicios de atención curativa alcanzó $85 \%$ de participación. Así, estas diferencias entre entidades federativas se reflejan en divergencias en las mejoras de los servicios de atención médica e infraestructura, ya que siguen existiendo lugares donde los individuos deben desplazarse a grandes distancias para poder recibir atención médica (Aguilera y Barraza-Lloréns, 2011). En este mismo sentido, Méndez (2019) expone que el indicador del gasto público per cápita se redujo, considerando el periodo 2010-2020, para todas las instituciones de salud. Esto ocurre, advierte, debido a la tendencia decreciente del monto destinado (como porcentaje del Producto Interno Bruto (PIB) del país), y a que la formulación presupuestaria no es acorde a la población que se espera atender, lo que provoca una fuerte desigualdad de los recursos asignados por institución.

El financiamiento público es uno de los elementos más importantes del sistema de salud, al reflejar la prioridad que el gobierno asigna a la atención en salud de la población (Gutiérrez, 2018). La figura 1 muestra la distribución geográfica del gasto público en salud per cápita total de 2017, agrupando a los estados conforme su nivel de gasto. Con el fin de considerar la heterogeneidad del gasto público al interior del país, se propone para el siguiente análisis la regionalización a partir de la figura 1 . 
Figura 1. Gasto público estatal en salud per cápita total, 2017

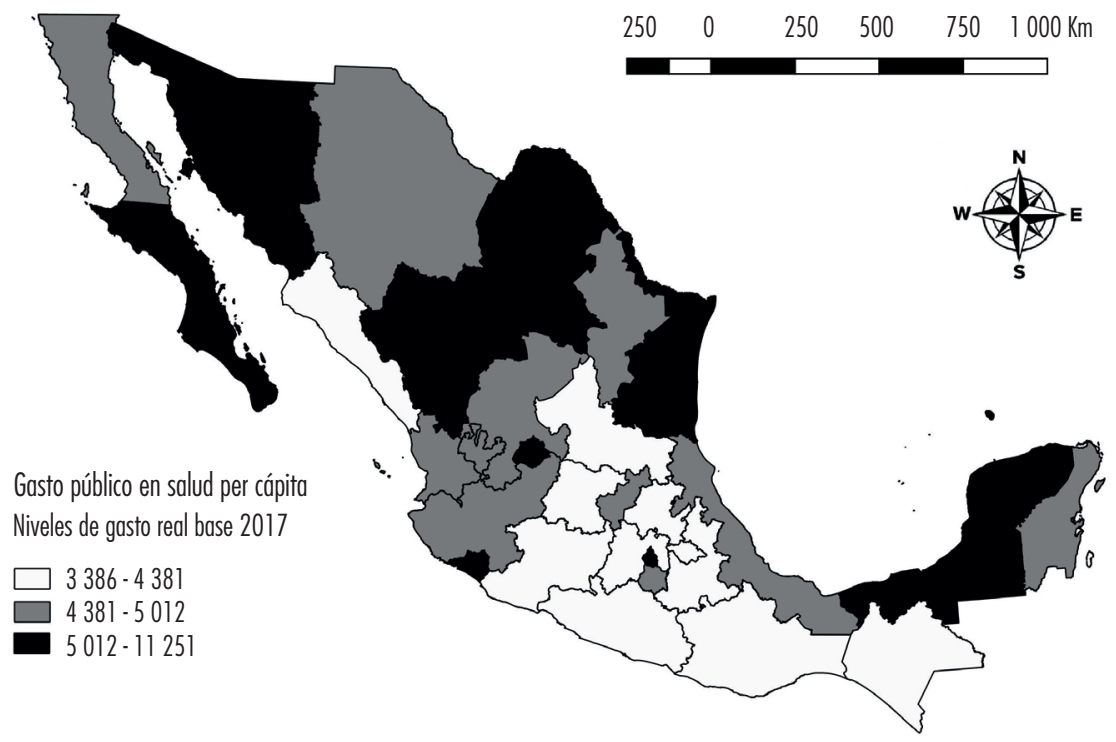

Fuente:elaboración propia a partir de SICUENTAS (2019).

- Región 1 (Gasto alto): Sonora, Coahuila, Tamaulipas, Baja California Sur, Durango, Aguascalientes, Colima, Ciudad de México, Tabasco, Campeche y Yucatán.

- Región 2 (Gasto medio): Baja California, Chihuahua, Nuevo León, Zacatecas, Nayarit, Jalisco, Querétaro, Veracruz, Morelos y Quintana Roo.

- Región 3 (Gasto bajo): Sinaloa, San Luis Potosí, Guanajuato, Michoacán, Hidalgo, México, Guerrero, Tlaxcala, Puebla, Guerrero, Oaxaca y Chiapas.

Lo antes descrito permite señalar que existen grandes desigualdades en la atención y en la calidad de los servicios de salud. Las diferencias se dan entre el sector público y el privado, pero además, dentro del público habrá diferencias entre sus instituciones (Martínez y Murayama, 2016), así como entre entidades federativas. 


\section{GASTOS CATASTRÓFICOS EN SALUD Y SU EFECTO EN LOS HOGARES}

El gasto catastrófico en salud ha sido un tema de estudio en México debido al impacto que tiene sobre el bienestar de los hogares. Los estudios sugieren que los gastos excesivos en salud se han reducido en el tiempo, lo cual se ha logrado en parte por el mayor acceso a servicios de salud, así como por el papel del programa público del sp. No obstante, los resultados muestran que existe heterogeneidad no sólo a nivel regional, sino también debido a las diferencias socioeconómicas de los individuos. A continuación se describen algunos documentos que sirven de fundamento para explorar esta problemática.

A partir de una encuesta desarrollada para examinar el impacto del sP, King et al. (2009) encuentran que este programa redujo el gasto catastrófico de los hogares. Asimismo, señalan que funciona mejor en zonas más pobres y se relaciona más con la cantidad y calidad de las instalaciones (centros de salud, hospitales, etcétera), que con las características de las familias. Por su parte, Grogger et al. (2014) subrayan que, a pesar de la reducción significativa de los gastos catastróficos, la protección del sp es heterogénea, ya que las áreas rurales con menor acceso a instalaciones de servicios de salud enfrentan reducciones menos significativas, al tiempo que zonas con mayor acceso a instalaciones más grandes sí presentan una considerable protección financiera. Lo anterior también se concluye en estudios como el de Leyva-Flores et al. (2013) y Gutiérrez et al. (2016), en los que se recalca la necesidad de contar con políticas integrales para atacar las desigualdades en el acceso a los servicios de salud.

Algunos estudios se centran en la búsqueda del efecto protector de los servicios sobre el gasto en salud en los diferentes rubros. Galárraga et al. (2010) apuntan a un efecto protector del sp a nivel nacional sobre el gasto en medicinas y gastos ambulatorios, pero no en gastos de hospitalización. Por su parte, Garrido-Latorre et al. (2008) analizan el porcentaje de recetas surtidas completamente a usuarios de los servicios ambulatorios de los servicios de la SSA y el sp para 2006, indicando que, si bien se había logrado mejorar el nivel de surtimiento completo de recetas, el catálogo de medicamentos privilegió los de bajo costo unitario a expensas de los de mayor precio y eficacia terapéutica. En un tenor similar, Wirtz et al. (2012) observan que la diferencia en la probabilidad de incurrir en gastos no es significativa entre los derechohabientes del sP y quienes no tienen afiliación, siendo estos últimos quienes presentan el mayor nivel de gasto. Lo anterior, explican, puede deberse al catálogo limitado de medicamentos aunado a su escasez; lo cual provoca que los hogares utilicen el servicio del sp, pero adquieran los medicamentos por su cuenta. 
El análisis de Pérez et al. (2013) se enfoca en la modalidad de servicios de atención médica en consultorios adyacentes a farmacias privadas. Confirman que el mayor uso es por parte de usuarios del sp, seguidos por quienes no tienen afiliación alguna. El problema de fondo es la gran diferencia en la probabilidad de obtener los medicamentos dependiendo de la institución que ofrece la atención, pues, respecto de 2006, la tasa de no-surtimiento se incrementó entre los usuarios de los servicios públicos (Wirtz et al., 2013). Un estudio más reciente de Pavón-León et al. (2017) refieren que, para los afiliados al sp en Xalapa, Veracruz, 95\% de la muestra tuvo que incurrir en gastos de bolsillo por medicamentos que estaban cubiertos por la institución.

Knaul et al. (2018) analizan el efecto del sp a partir de la información de la ENIGH, de 2004 a 2012. Los autores encuentran que la afiliación al sp reduce la probabilidad de incurrir en gastos catastróficos, así como la presencia del SP en la entidad y la ampliación en su cobertura. Añaden que, si en el hogar viven adultos mayores y menores de cinco años, entonces se incrementa la probabilidad de presentar gastos excesivos, lo mismo ocurre si el hogar recibe remesas. A pesar de lo anterior, los afiliados al sp y los no afiliados a ninguna institución no presentan diferencias significativas entre los niveles de gasto catastrófico. Con la ENIGH 2010, Díaz-González y Ramírez-García (2017) evidencian que, si bien una causa del gasto en salud es el no estar afiliado al sistema público, el tener SP tampoco es garantía de no presentarlo. Apuntan que el aseguramiento es un determinante del gasto, pero no lo es en la probabilidad de incurrir en gastos catastróficos, ya que más del $90 \%$ del gasto en salud proviene del bolsillo de las familias. Asimismo, el ingreso no parece ser determinante en la presencia de gastos catastróficos, pues es un fenómeno que se presenta a lo largo de la distribución.

De la revisión de literatura es posible desprender tres conclusiones generales respecto del gasto en salud en México. Primero, la fragmentación del sistema de salud provoca diferencias en términos de la cobertura, así como el nivel de gasto de bolsillo sobre toda la población. Segundo, el hecho de que el sp funcione a partir de la infraestructura existente reduce sus posibilidades de proveer adecuadamente a sus beneficiarios. Tercero, en la mayoría de los documentos se concluye que el comportamiento del gasto de bolsillo es heterogéneo entre la población que acude a los diversos sistemas; en particular, el SP es el que presenta los menores efectos protectores, además de ser altamente diferenciado, según las características socioeconómicas y regionales de los individuos. 


\section{DATOS Y MODELOS EMPLEADOS}

Según los reportes realizados por la Organización Mundial de la Salud (Organización Mundial de la Salud [oms], 2017) no existe un único enfoque para medir los gastos catastróficos en salud. Para los ods el gasto en salud es posible definirlo como catastrófico cuando supera el 10 o $25 \%$ de los ingresos o del consumo total (oMs, 2017), esta metodología suele denominarse "participación en el presupuesto". En el presente estudio se emplea dicha perspectiva considerando el umbral del $10 \%$ sobre el ingreso, tomando en cuenta que el ingreso involucra transferencias gubernamentales y remesas del extranjero, montos relevantes que se destinan hacia la salud (Díaz-González y RamírezGarcía, 2017; Knaul et al., 2018; Mora-Rivera et al., 2018). Esto además con fines comparativos respecto de otros estudios realizados para México, los cuales emplean el enfoque de Xu et al. (2003), que consideran la capacidad de pago (ingreso, o consumo, menos el gasto en alimentación) de los hogares.

Todas las variables manejadas en el presente documento se obtienen o construyen a partir de la ENIGH 2018 (véase tabla 2), y se sugieren a partir de la revisión de literatura nacional e internacional sobre gasto de bolsillo y gasto catastrófico (véase Guzmán, 2018).

En el cálculo del gasto en salud se involucran los 72 rubros considerados en la ENIGH 2018 para obtener el gasto en salud corriente total mensual per cápita (lo cual se denominará gasto de bolsillo, $G B$ ). Se estiman dos modelos tobit, uno para el gasto de bolsillo y uno para el gasto de bolsillo catastrófico $(G B C)$. Es decir, en el segundo modelo la población que presente $G B$, pero que éstos no sean excesivos, tendrá en la variable dependiente el valor de cero; de manera que la distribución de los $G B C$ será aproximadamente continua para los valores en el que el gasto en salud es positivo y catastrófico. La decisión de realizar una comparación se debe al planteamiento que establece que destinar un mayor monto de ingreso al gasto en salud puede ser visto como una forma de inversión de los hogares, y no como un problema, a menos que dicho gasto represente una dificultad financiera (Dmytraczenko et al., 2017).

El modelo tobit se representa en función de una variable latente determinada por las variables independientes observadas y el término del error que se supone presenta una distribución normal (Johnston y Dinardo, 1997; Greene, 2003; Bravo y Vásquez, 2008). La variable observada será igual a la variable latente cuando esta variable sea positiva (en este caso cuando el individuo incurra en $G B$ y en $G B C$, respectivamente). Los parámetros $\beta$ estimados miden de forma directa los efectos marginales de las variables independientes sobre la variable del gasto en salud. 
Hada M. Sáenz-Vela y Ángela M. Guzmán-Giraldo

Tabla 2. Definición de las variables

\begin{tabular}{|c|c|}
\hline Variable & Definición \\
\hline \multicolumn{2}{|c|}{ Variables regionales } \\
\hline REGION & $\begin{array}{l}\text { Región en la que reside el individuo, según regionalización de la figura 1. Región 1: Gasto alto; } \\
\text { Región 2: Gasto medio; Región 3: Gasto bajo (categoría de referencia). }\end{array}$ \\
\hline RURAL & Dicotómica, 1 si el hogar está ubicado en una localidad con menos de 2500 habitantes. \\
\hline \multicolumn{2}{|c|}{ Características del hogar } \\
\hline ADMAY & Binaria, 1 si en el hogar hay personas con edad mayor 0 igual a 65 años. \\
\hline NINOS & Binaria, 1 si en el hogar hay niños menores de cinco años. \\
\hline OCUPA & Número de ocupados dentro del hogar. \\
\hline \multicolumn{2}{|c|}{ Características del individuo } \\
\hline SP & Binaria, 1 si el individuo se encuentra inscrito en el programa SP. \\
\hline IMSS-ISSSTE & Binaria, 1 si el individuo se encuentra inscrito al IMSS o al ISSSTE (federal 0 estatal). \\
\hline OTRO & $\begin{array}{l}\text { Binaria, } 1 \text { si el individuo se encuentra inscrito al servicio provisto por PEMEX, SEDENA, SEMAR, IMSS-P, } \\
\text { privados u otros. }\end{array}$ \\
\hline DISCAP & Binaria, 1 si el individuo presenta alguna discapacidad. \\
\hline EDAD & Edad del individuo. \\
\hline SEXO & Binaria, 1 si el individuo es hombre. \\
\hline PRIVADO & $\begin{array}{l}\text { Binaria, } 1 \text { si el individuo acudió a un servicio privado de salud la última vez que se atendió por algún } \\
\text { malestar. }\end{array}$ \\
\hline ICTPC & Ingreso corriente total mensual per cápita. ${ }^{a}$ \\
\hline QUINTIL & $\begin{array}{l}\text { Indica el quintil en el que se encuentra el individuo de acuerdo con su ingreso corriente total mensual } \\
\text { per cápita, se tomará como referencia el primer quintil. }{ }^{b}\end{array}$ \\
\hline \multicolumn{2}{|c|}{ Variables dependientes } \\
\hline GB & Gasto de bolsillo en salud mensual per cápita. ${ }^{c}$ \\
\hline GBC & Si GB/ICTPC $\geq(0.10)^{\star}(I C T P C)$ entonces $G B C=G B / I C T P C$, en caso contrario $G B C=0$. \\
\hline
\end{tabular}

Notas: ${ }^{a}$ se considera el ingreso corriente total per cápita que emplea el coneval para el cálculo de la pobreza. Para la depuración de la base de datos, se eliminaron las observaciones sin reporte, reporte de cero y reportes de menos de 40 y de más de 50 mil pesos mensuales en la variable ingreso corriente total per cápita, lo que significó la pérdida de menos del 1\% de la muestra total. ${ }^{b}$ A la variable ICTPC se le aplica una transformación Box-Cox para normalizar la distribución de los datos, a partir de los datos transformados se realizó el análisis de quintiles. 'El gasto en cuidados de la salud se conforma de tres conjuntos: atención ambulatoria, hospital y medicinas, y considera el gasto en los últimos tres meses. El gasto en salud mensual se divide entre el número de adultos equivalentes que conforman el hogar, considerando la escala de CONEVAL para el cálculo de pobreza.

Fuente: elaboración propia a partir de la ENIGH 2018. 


\section{ANÁLISIS CONTEXTUAL Y DE RESULTADOS EMPÍRICOS}

Además de la información sobre el gasto en salud en los últimos tres meses, se reporta en la ENIGH 2018 información sobre el uso de servicios de salud, así como su afiliación. A los individuos se les pregunta cuándo fue la última vez que sufrió algún dolor, malestar, enfermedad o accidente que le impidiera realizar sus actividades cotidianas y en cuál institución médica recibió atención médica.

En la figura 2a se muestra un análisis de la distribución de la población por quintiles según lugar de afiliación. Se desprende que, ante incrementos en el ingreso, disminuye la cantidad de población afiliada al sp; lo contrario ocurre con la afiliación al IMSS y al ISSSTE. Los resultados anteriores son consistentes con los hallazgos de Méndez (2017), quien encuentra que los beneficiarios del sp están concentrados en la población de menores ingresos (deciles de I-V) y los adscritos a las instituciones formales (IMSS e ISSSTE) pertenecen a los deciles más altos (VI-X). En la figura $2 \mathrm{~b}$ se presenta la distribución del servicio utilizado la última vez que se atendió, de acuerdo con el quintil en que se ubican. Dicho resultado es consistente con otros estudios realizados (DanesedlSantos et al., 2011; Ávila-Burgos et al., 2013; Bautista-Arredondo et al., 2014), pues conforme se eleva el nivel socioeconómico de los individuos, es menos probable que acudan al servicio de salud al cual se encuentran afiliados; ya que la asistencia a la servicios provistos por la SSA se concentra en la población más pobre, al tiempo que la población de mejores ingresos se inclina por servicios privados (la categoría Otros refiere a servicios de consultorios y hospitales privados, así como alternativos). No obstante, en este último punto se destaca que, incluso en el quintil de menor ingreso, se reporta una asistencia a servicios privados alta.

La tabla 3 presenta información sobre el $G B$ en salud mensual per cápita (sólo considerando a individuos que reportaron gasto positivo), de acuerdo con el servicio al que estén afiliados. El mayor gasto promedio en salud lo presentaron los afiliados al ISSSTE, seguido por los adscritos a PEMEX. Se observa también que los individuos del quintil más bajo afiliados al SP y al IMSs-P también presentan $G B$, en ambos casos ligeramente menores a lo reportado por los del Imss o el Issste. No obstante, los afiliados al sp del primer quintil llegaron a reportar gastos máximos mayores a los incurridos por los inscritos a los otros servicios de salud. 
2 븅

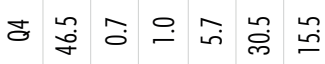
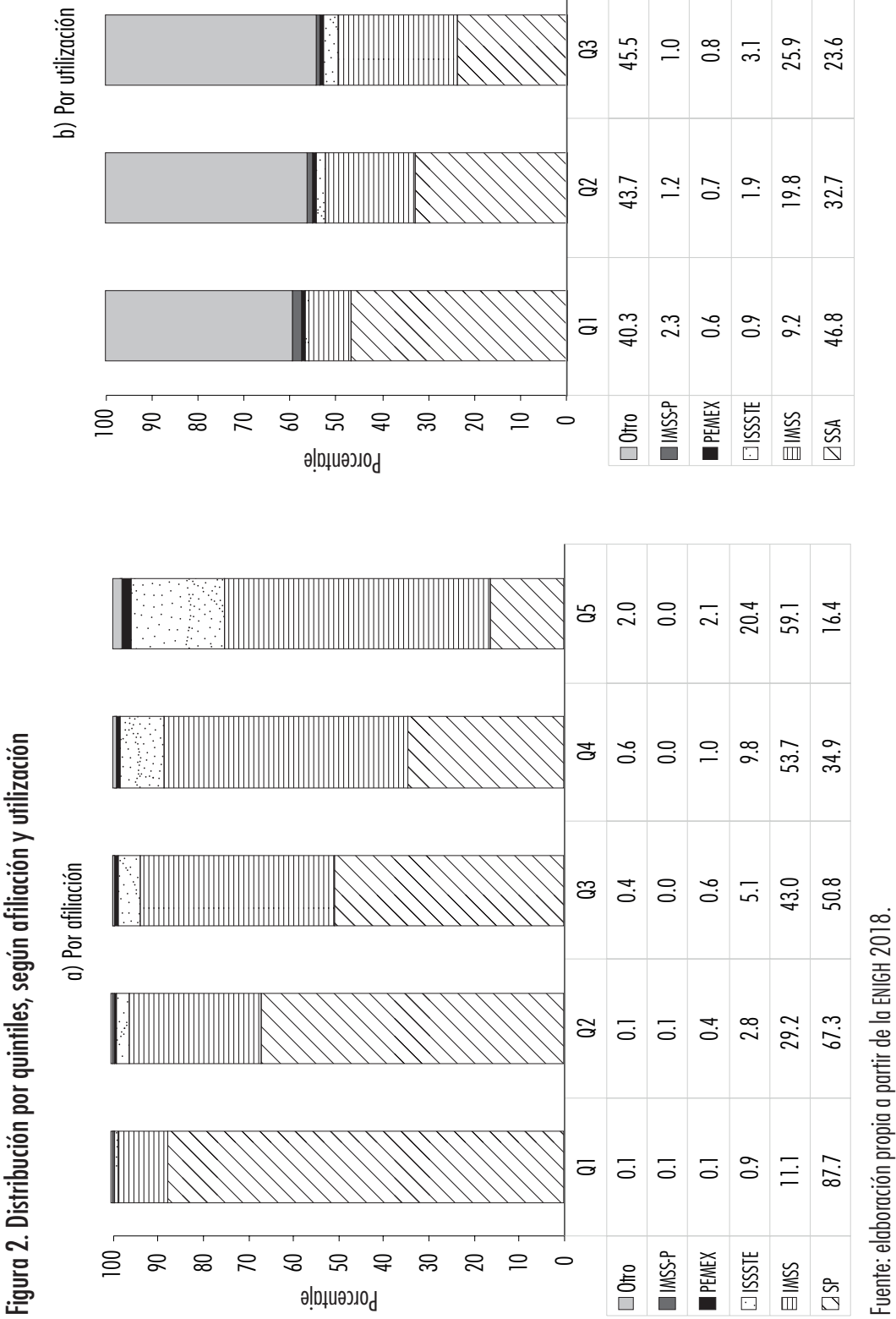
Tabla 3. Máximo y media del GB por quintil y por institución

\begin{tabular}{llrrrrr}
\hline & \multicolumn{5}{c}{ Quintil } \\
\hline Institución & & \multicolumn{1}{c}{1} & \multicolumn{1}{c}{2} & \multicolumn{1}{c}{3} & \multicolumn{1}{c}{4} & \multicolumn{1}{c}{5} \\
\hline SP & Máximo & 6075.69 & 22150.63 & 8197.26 & 30499.90 & 25173.78 \\
& Media & 63.65 & 74.21 & 102.65 & 150.51 & 314.06 \\
IMSS & Máximo & 3114.95 & 22150.63 & 6913.04 & 24667.87 & 46956.51 \\
& Media & 75.00 & 77.58 & 82.73 & 119.45 & 294.95 \\
ISSSTE & Máximo & 1159.09 & 1388.07 & 8197.26 & 5959.19 & 17253.25 \\
& Media & 81.95 & 81.21 & 113.15 & 146.67 & 365.39 \\
PEMEX & Máximo & 177.06 & 729.55 & 688.57 & 616.91 & 7628.91 \\
& Media & 51.64 & 50.41 & 94.62 & 75.10 & 232.76 \\
IMSS-P & Máximo & 179.02 & 784.07 & 131.87 & 101.64 & 582.82 \\
& Media & 59.33 & 109.91 & 33.24 & 17.69 & 181.58 \\
\hline
\end{tabular}

Fuente: elaboración propia a partir de la ENIGH 2018.

También puede analizarse el comportamiento de los gastos catastróficos en particular. De acuerdo con la tabla 4, los gastos catastróficos afectan principalmente a las personas ubicadas en el primer quintil y que habitan en las regiones con gasto público per cápita medio y bajo (Región 2 y 3 , respectivamente). Aunque no se omite señalar que los gastos excesivos parecen presentarse a lo largo del territorio, aún ante un mayor gasto público en salud. Si bien las diferencias entre regiones no son considerablemente grandes, se percibe que sí se mantienen a lo largo de la distribución dentro de una misma región (excepto para el quintil más bajo).

En cuanto a los resultados de los diferentes modelos econométricos propuestos se presentarán aquellos que tuvieron los mejores ajustes. Las variables explicativas (presentadas en la tabla 2) fueron incluidas de acuerdo con la revisión de literatura nacional e internacional sobre el tema (Séne y Cissé, 2015; Lam et al., 2016; Anderson et al., 2017; López del Amo et al., 2018); además de los mencionados en secciones anteriores. Todos los coeficientes resultaron significativos de manera individual, y se encontró una buena significancia total del modelo (Prob>chi2) en el modelo tobit. Dado que los resultados no se interpretan directamente, se muestran en la tabla 5 los efectos marginales de la variable censurada del gasto en salud. En la segunda y tercera columna se muestran la incidencia y la probabilidad de incurrir en $G B$ positivos, señala- 
Hada M. Sáenz-Vela y Ángela M. Guzmán-Giraldo

Tabla 4. Porcentaje de incidencia de gasto catastrófico por regiones

\begin{tabular}{lccc}
\hline Quintil & Región 1 & Región 2 & Región 3 \\
\hline 1 & 7.2 & 11.7 & 10.3 \\
2 & 3.6 & 5.5 & 4.4 \\
3 & 3.2 & 4.4 & 4.4 \\
4 & 3.3 & 3.1 & 4.4 \\
5 & 4.1 & 4.1 & 4.8 \\
\hline Total & 4.0 & 5.3 & 5.8 \\
\hline
\end{tabular}

Fuente: elaboración propia a partir de la ENIGH 2018.

dos como $\operatorname{Pr}(G B>0)$ y $\operatorname{Pr}(G B=0)$, respectivamente; y de manera análoga se reportan la incidencia y la probabilidad de incurrir en gastos catastróficos en la cuarta y quinta columna.

Se encuentran algunas diferencias interesantes entre el comportamiento del $G B$ y el $G B C$. Respecto de la región de menor gasto público per cápita, los individuos que habitan en estados de la región con niveles medios y altos de gasto público tienen menores niveles de incidencia de $G B$ y de $G B C$, así como reducciones en la probabilidad de su ocurrencia. No obstante, la incidencia de los $G B C$ se incrementa en $17.5 \%$ para aquellos individuos que habitan en zonas rurales. De esta forma, se verifica que el gasto público puede favorecer la protección financiera, por lo que es importante retomar la inversión del Estado en este rubro.

Asimismo, la presencia de menores de cinco años incrementa los gastos de bolsillo, así como los gastos catastróficos. Mientras que la presencia de adultos mayores en un hogar pueden elevar la incidencia de gastos catastróficos hasta en $23.87 \%$. El número de ocupados en el hogar tiene un efecto positivo sobre el $G B$, lo cual potencialmente puede asociarse a la idea del gasto en salud como una inversión y ayuda a reducir la incidencia del gasto catastrófico en 5.70\%.

En cuanto a las características del individuo es innegable que el acudir a servicios privados multiplicó los gastos en los hogares. Se observan incrementos en la incidencia de $G B$ y $G B C$ que rondan en $50 \%$, al tiempo que incrementa la probabilidad de incurrir en $G B$ en $16.81 \%$, y en $G B C$ en $3.62 \%$. Si el individuo padece alguna discapacidad, los $G B$ se elevan en $17.98 \%$ y los $G B C$ hasta en $29.44 \%$. Los hombres presentan menores montos, para ambos tipos de gasto; mientras que la edad tiene un efecto positivo, aunque marginal, sobre los excesivos. 
Tabla 5. Efectos marginales sobre el nivel de gasto de bolsillo y catastrófico

\begin{tabular}{|c|c|c|c|c|}
\hline & \multicolumn{2}{|c|}{ Gasto de bolsillo } & \multicolumn{2}{|c|}{ Gasto catastrófico } \\
\hline & $\operatorname{Pr}(G B>0)$ & $\operatorname{Pr}(G B=0)$ & $\operatorname{Pr}(G B C>0)$ & $\operatorname{Pr}(G B C=0)$ \\
\hline \multicolumn{5}{|l|}{ Variables regionales } \\
\hline REGION I (Gasto alto) & $-0.1125^{\star \star \star}$ & $-0.0323^{\star \star \star}$ & $-0.1391^{\star \star \star}$ & $-0.0106^{\star \star \star}$ \\
\hline REGION 2 (Gasto medio) & $-0.1523^{\star \star \star}$ & $-0.0437^{\star \star \star}$ & $-0.1377^{\star \star \star}$ & $-0.0105^{\star \star \star}$ \\
\hline RURAL & & & $0.1750^{\star \star \star}$ & $0.0133^{\star * \star}$ \\
\hline \multicolumn{5}{|l|}{ Características del hogar } \\
\hline ADMAY & $0.0995^{\star \star \star}$ & $0.0285^{\star \star \star}$ & $0.2387^{\star \star \star}$ & $0.0182^{\star \star \star}$ \\
\hline NINOS & $0.0991^{\star \star *}$ & $0.0284^{\star \star \star}$ & $0.0436^{\star \star \star}$ & $0.0033^{\star \star \star}$ \\
\hline OCUPA & $0.0640^{\star \star \star}$ & $0.0184^{\star \star \star}$ & $-0.0571^{\star \star \star}$ & $-0.0044^{\star \star \star}$ \\
\hline \multicolumn{5}{|l|}{ Características del individuo } \\
\hline SP & $0.0734^{\star \star \star}$ & $0.0211^{\star \star \star}$ & $0.0385^{\star}$ & $0.0029^{*}$ \\
\hline IMSS-ISSSTE & $-0.0398^{\star \star \star}$ & $-0.0114^{\star \star \star}$ & $-0.1095^{\star \star \star}$ & $-0.0084^{\star \star \star}$ \\
\hline OTRO & $-0.0973^{\star \star \star}$ & $-0.0279^{\star \star \star}$ & $-0.2314^{\star \star \star}$ & $-0.0177^{\star \star \star}$ \\
\hline DISCAP & $0.1798^{\star \star \star}$ & $0.0516^{\star \star \star}$ & $0.2943^{\star \star \star}$ & $0.0225^{\star \star \star}$ \\
\hline EDAD & $-0.0004^{\star \star}$ & $-0.0001^{\star \star}$ & $0.0050^{\star \star \star}$ & $0.0004^{\star \star \star}$ \\
\hline SEXO & 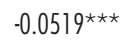 & $-0.0149^{\star \star \star}$ & $-0.0555^{\star \star \star}$ & $-0.0042^{\star \star \star}$ \\
\hline PRIVADO & $0.5856^{\star \star \star}$ & $0.1681^{\star \star \star}$ & $0.4741^{\star \star \star}$ & $0.0362^{\star \star \star}$ \\
\hline QUINTIL 2 & $0.1181^{\star \star \star}$ & 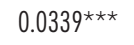 & $-0.4165^{\star \star \star}$ & $-0.0318^{\star \star \star}$ \\
\hline QUINTIL 3 & $0.2481^{\star \star \star}$ & $0.0712^{\star \star \star}$ & $-0.4044^{\star \star \star}$ & $-0.0309^{\star \star \star}$ \\
\hline QUINTIL 4 & $0.3660^{\star \star \star}$ & $0.1050^{\star \star \star}$ & $-0.3823^{\star \star \star}$ & $-0.0292^{\star \star \star}$ \\
\hline QUINTIL 5 & $0.6380^{\star \star \star}$ & $0.1831^{\star \star \star}$ & $-0.3430^{\star \star \star}$ & $-0.0262^{\star \star \star}$ \\
\hline
\end{tabular}

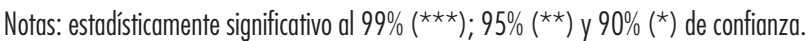

Fuente: elaboración propia a partir de la ENIGH 2018. 
Es necesario destacar el efecto del tipo de servicio de salud al que se tiene afiliación, considerando como punto de referencia no contar con ningún servicio. Pues aquellos individuos inscritos en el programa sP presentan mayores incidencias de $G B$ respecto de quienes no tienen afiliación, aunque son apenas significativas para el caso del $G B C$. Mientras que la inscripción al IMSS y al ISSSTE reducen esas mismas incidencias, alcanzando una disminución de casi $10 \%$ en el caso de $G B C$. Se observan reducciones todavía mayores en el caso de afiliación a servicios como Pemex o privados, alcanzando $23.14 \%$ para los $G B C$. El panorama no es muy diferente al considerar las probabilidades de incurrir en $G B C$. Estar afiliado al sP incrementa la probabilidad -aunque marginal y apenas significativa respecto de no tener adscripción alguna-, de presentar $G B C$. Las afiliaciones a servicios públicos ayudan a reducir ligeramente dicha probabilidad.

El nivel de ingresos de los individuos también presenta un efecto importante. Los resultados sugieren que conforme se cuenta con mayores ingresos, se tiene una tendencia a incrementar el gasto en salud. Por ejemplo, los individuos del quintil más alto elevan su $G B$ en $63.52 \%$, respecto del quintil 1 . En el caso de los $G B C$, se percibe que el ingreso permite reducir la probabilidad de padecerlos; no obstante, conforme se incrementa el nivel de ingresos, esta probabilidad se reduce ligeramente. Esto último remite a lo visto en la estadística descriptiva (véanse tablas y figuras previas), así como la anotación de DíazGonzález y Ramírez-García (2017), quienes señalan que los gastos excesivos son un problema que se padece a lo largo de la distribución.

\section{REFLEXIONES FINALES}

Los problemas de salud no son exclusivos de algún segmento de la población y los gastos excesivos se presentan en cualquier nivel de ingreso; sin embargo, en México se pretende trabajar la dimensión salud desde una perspectiva de derechos y justicia social para combatir la pobreza (CONEval, 2009). Bajo este enfoque, el acceso debe de darse a cualquier nivel de ingreso y ajustarse a las diversas necesidades de servicio, financiamiento y localización de recursos. Para que el acceso permita prevenir enfermedades que se asocian, comúnmente, a situaciones de gasto catastrófico, sobre todo, en sectores de la población en desventaja.

El ejercicio empírico realizado ofrece evidencia del comportamiento sobre el gasto en salud en función de ciertas características de las personas. Se observó que las variables regionales, del hogar y de los individuos, siguen siendo 
fuentes de diferenciación para el $G B$ y $G B C$; incrementándose el gasto en salud en las zonas rurales, ante la presencia de niños, adultos mayores y personas con alguna discapacidad en el hogar, así como ante aumentos en el nivel de ingreso individual. Sumado a lo anterior, se verificó que el gasto público en salud genera diferencias entre los niveles de gasto privado, impactando particularmente a los individuos del primer quintil de ingresos.

Una considerable parte de la literatura se enfoca en verificar si existe un efecto protector del sp, y se debe a que gran parte de la población más desfavorecida se encontraba cubierta por dicho esquema. Los resultados aquí expuestos permiten concluir que el sp logró proteger a sus afiliados, aunque de manera diferenciada; siendo precisamente esa una de las razones por las que la afiliación al sp se volvió un elemento que aumentaba la probabilidad de incurrir en gastos catastróficos. Una de las críticas al esquema del sp fue, precisamente, que incrementó la demanda por servicios, al tiempo que no se lograron ofrecer servicios suficientes y de calidad. Es importante recordar que las decisiones sobre el financiamiento del sp respondían, predominantemente, al número de afiliados y no a la capacidad instalada, lo cual amplió las brechas entre las entidades federativas (Flamand y Moreno-Jaimes, 2015) e implicó la necesidad de una revisión detallada sobre el funcionamiento de este programa presupuestal.

El aumento en la demanda de servicios de salud se da en un escenario en el que son los hogares más pobres y de zonas rurales los que presentan mayores tasas de gasto en salud. Soto-Estrada et al. (2016) apuntan que en los últimos años se generaron cambios importantes en el comportamiento epidemiológico de las enfermedades, aunado a las modificaciones en la composición del hogar, debido a los cambios ambientales, económicos, sociales y de transición demográfica. Mientras que en el periodo 2008-2018, el grupo de menores de 15 años pasó de $34.1 \%$ del total de la población a $25.3 \%$, el grupo de 60 y más años pasó de 7.3 a 12.3\% (INEGI, 2019).

Una mayor concentración de habitantes en edades avanzadas está fuertemente relacionada con el gasto en salud. Salinas-Escudero et al. (2019) y Granados-Martínez y Nava-Bolaños (2019) analizan los factores que influyen en el $G B$ y $G B C$ de los adultos mayores en el país, respectivamente. El primer estudio encuentra que los medicamentos fueron el mayor componente de los gastos de bolsillo, pues oscila entre 75 y $90 \%$ del gasto total reportado en salud. El segundo estudio resalta que los factores con mayor impacto sobre los gastos catastróficos son la presencia de algún miembro discapacitado en el hogar, lo que incrementa los gastos, y la afiliación a las instituciones, con un efecto opuesto. 
Para 2017, Pérez-Cuevas y Doubova (2019) señalan que cuatro de cada diez individuos padecían una enfermedad crónica, lo que se agrega a las de por sí existentes barreras financieras y de transporte que incrementan los gastos de bolsillo. Estos autores destacan también las deficiencias en la calidad de la atención y la escasa oferta de servicios preventivos para enfermedades crónicas. En este sentido, Soto-Estrada et al. (2016) subrayan la importancia de la prevención de enfermedades y promoción de la salud, pues en México alrededor del 30\% de las muertes se concentran en tres principales enfermedades: diabetes mellitus tipo 2 , enfermedades isquémicas del corazón y enfermedades cerebro-vasculares; siendo la diabetes la principal causa de muerte en el país, y a su vez, una importante causa de la demanda de atención médica y de hospitalización. Además, indican, estos padecimientos comparten entre sí factores de riesgo que pudieran ser prevenibles, como son el sobrepeso, la obesidad, el colesterol, el tabaquismo y la hipertensión arterial.

Señalan Dávila-Torres et al. (2015) que, de acuerdo con estimaciones de la Organización para la Cooperación y el Desarrollo Económicos (OCDE), una estrategia de prevención efectiva evitaría alrededor de 55 mil muertes por enfermedades crónicas en México. No obstante, a pesar de la existencia de un gran número de enfermos crónicos en el país, ello no se ha traducido en mayores acciones de atención preventiva (Pérez-Cuevas y Doubova, 2019). Por lo tanto, es importante que el sistema de salud evolucione frente a los nuevos retos que las sociedades enfrentan, en términos de la transición demográfica y epidemiológica, para mejorar las condiciones en las que se puede soportar una emergencia sanitaria, como la actual pandemia Covid-19.

\section{BIBLIOGRAFÍA}

Aguilera, N. y Barraza-Lloréns, M. (2011). FAssA: análisis sobre equidad y alternativas de asignación. En C. Chiapa y C. Velázquez (coord.). Estudios del ramo 33 (p. 115). CONEVAL.

Anderson, G., Ilcisin, L., Kayima, P., Abesiga, L., Portal-Benitez, N., Ngonzi, J., Ronald, M. y Shrime, M. (2017). Out-of-pocket payment for surgery in Uganda: The rate of impoverishing and catastrophic expenditure at a government hospital. PLoS ONE, 12(10). https://dx.doi. org/10.1371\%2Fjournal.pone.0187293

Ávila-Burgos, L., Serván-Mori, E., Wirtz, V., Sosa-Rubí, S. y Salinas-Rodríguez, A. (2013). Efectos del Seguro Popular sobre el gasto en salud en 
hogares mexicanos a diez años de su implementación. Salud Pública de México, 55(S2). http://dx.doi.org/10.21149/spm.v55s2.5103

Baird, K. (2016). The incidence of high medical expenses by health status in seven developed countries. Health Policy, 120(1). https://doi.org/10.1016/j. healthpol.2015.10.004

Bautista-Arredondo, S., Serván-Mori, E., Colchero, A., Ramírez-Rodríguez, B. y Sosa-Rubí, S. (2014). Análisis del uso de servicios ambulatorios curativos en el contexto de la reforma para la protección universal en salud en México. Salud Pública de México, 56(1). https://doi.org/10.21149/spm. v56i1.7319

Bravo, D. y Vásquez, J. (2008). Curso: Microeconometría aplicada. Departamento de Economía. Universidad de Chile. https://bit.ly/3fiW3CQ

Cinaroglu, S. (2018). Demographic and welfare state predictors of outof-pocket health expenditures: A path analytic model. Journal of Social Service Research, 44(4). https://doi.org/10.1080/01488376.2018.1477 703

Consejo Nacional de Evaluación de la Política de Desarrollo Social (CONEval) (2009). Metodología para la medición multidimensional de la pobreza en México. Coneval.

(2019). Anexo estadístico de pobreza en México 2018. https://bit. ly/2KwrlLn

Danese-dlSantos, L., Sosa-Rubí, S. y Valencia-Mendoza, A. (2011). Analysis of changes in the association of income and the utilization of curative health services in Mexico between 2000 and 2006. BMC Public Health, 11(771). https://doi.org/10.1186/1471-2458-11-771

Dávila-Torres, J., González-Izquierdo, J. y Barrera-Cruz, A. (2015). Panorama de la obesidad en México. Revista Médica del Instituto Mexicano del Seguro Social, 53(2). https://bit.ly/2UOine2

Díaz-González, E. y Ramírez-García, J. (2017). Gastos catastróficos en salud, transferencias y remesas en México. Papeles de Población, 23(91). https:// rppoblacion.uaemex.mx/article/view/8247

Dmytraczenko, T., Montenegro, F. y Aten, A. (2017). Políticas de cobertura universal en salud en América Latina y el Caribe. En T. Dmytraczenko y G. Almeida (eds.). Hacia la cobertura universal en salud y la equidad en América Latina y el Caribe (pp. 57-88). Banco Mundial. http://dx.doi. org/10.1596/978-1-4648-1177-7

Encuesta Nacional de Ingresos y Gastos de los Hogares (ENIGH) (2018). Nueva serie. https:/www.inegi.org.mx/programas/enigh/nc/2018/ 
Flamand, L. y Moreno-Jaimes, C. (2015). La protección social en salud durante el gobierno de Calderón. Avances y rezagos en el diseño y la implementación del Seguro Popular (2006-2012). Foro Internacional, 55(1). https://doi.org/10.24201/fi.v55i1.2266

Galárraga, O., Sosa-Rubí, S., Salinas-Rodríguez, A. y Sesma-Vázquez, S. (2010). Health insurance for the poor: Impact on catastrophic and outof-pocket health expenditures in Mexico. The European Journal of Health Economics, 11(5). https://dx.doi.org/10.1007\%2Fs10198-009-0180-3

García-Junco, D. (2012). La transformación del sistema de salud y el Seguro Popular. Gaceta Médica de México, 148(6). https://bit.ly/36WrG1m

Garrido-Latorre, F., Hernández-Llamas, H. y Gómez-Dantes, O. (2008). Surtimiento de recetas a los afiliados al Seguro Popular de México. Salud Pública de México, 50(S4). https://saludpublica.mx/index.php/spm/article/view/4855

Gómez-Dantés, O., Sesma, S., Becerril, V., Knaul, F., Arreola, H. y Frenk, J. (2011). Sistema de Salud de México. Salud Pública de México, 53(S2). https://saludpublica.mx/index.php/spm/article/view/5043

Granados-Martínez, A. y Nava-Bolaños, I. (2019). Gastos catastróficos por motivos de salud y hogares con personas mayores en México. Papeles de población, 25(99). https://rppoblacion.uaemex.mx/article/view/10279

Greene, W. (2003). Econometric Analysis. Prentice Hall.

Grogger, J., Arnold, T., León, A. y Ome, A. (2014). Heterogeneity in the effect of public health insurance on catastrophic out-of-pocket health expenditures: The case of Mexico. Health Policy and Planning, 30(5). https:// doi.org/10.1093/heapol/czu037

Gutiérrez, C. (2018). El enfoque sistémico para la priorización en salud. En U. Giedion, M. Distrutti, A. L. Muñoz, D. Pinto y A. M. Díaz (eds.). La priorización en salud paso a paso: cómo articulan sus procesos México, Brasily Colombia (pp. 5-14). Banco Interamericano de Desarrollo. http://dx.doi. org/10.18235/0001092

Gutiérrez, J., García-Saisó, S., Espinosa-de la Peña, R. y Balandrán, D. (2016). Monitoreo de la desigualdad en protección financiera y atención a la salud en México: análisis de las encuestas de salud 2000, 2006 y 2012. Salud Pública de México, 58(6). http://dx.doi.org/10.21149/spm.v58i6.7920

Guzmán, Á. (2018). Gastos catastróficos en salud. Un análisis de sus determinantes y su relación con la pobreza, México 2016 [Tesis de Maestría, Universidad Autónoma de Coahuila]. www.cise.uadec.mx/downloads/tesis/ Maestria2016-2018_GGAM.PDF 
Instituto Nacional de Estadística y Geografía (INEGI) (2018). Resultados de la Encuesta Nacional de Ocupación y Empleo. Cifras durante el tercer trimestre de 2018. https://bit.ly/36YVlqu

(2019). Estadísticas a propósito del Día Mundial de la Población. https://bit.ly/2ULtggL

Johnston, J. y Dinardo, J. (1997). Econometric Models. McGraw-Hill.

King, G., Gakidou, E., Imai, K., Lakin, J., Moorre, R., Nall, C., Ravishankar, N., Vargas, M., Téllez-Rojo, M., Hernández, J., Hernández, M. y Hernández, H. (2009). Public policy for the poor? A randomised assessment of the Mexican universal health insurance programme. The Lancet, 373 (9673). https://doi.org/10.1016/S0140-6736(09)60239-7

Knaul, F., Arreola-Ornelas, H., Wong, R., Lugo-Palacios, D. y Méndez-Carniado, Ó. (2018). Efecto del Seguro Popular de Salud sobre los gastos catastróficos y empobrecedores en México, 2004-2012. Salud Pública de México, 60(2). https://doi.org/10.21149/9064

Lam, C., Guo, V., Wong, C., Yu, E. y Fung, C. (2016). Poverty and health related quality of life of people living in Hong Kong: Comparison of individuals from low income families and the general population. Journal of Public Health, 39(2). https://doi.org/10.1093/pubmed/fdw046

Leyva-Flores, R., Infante-Xibille, C., Gutiérrez, J. y Quintino-Pérez, F. (2013). Inequidad persistente en salud y acceso a los servicios para los pueblos indígenas de México, 2006-2012. Salud Pública de México, 55(S2). https:// doi.org/10.21149/spm.v55s2.5107

López del Amo, M., Benítez, V. y Martín-Martín, J. (2018). Long term unemployment, income, poverty, and social public expenditure, and their relationship with self-perceived health in Spain (2007-2011). BMC Public Health, 18(133). https://doi.org/10.1186/s12889-017-5004-2

Martínez, J. y Murayama, C. (2016). El sistema de atención a la salud en México. En C. Murayama y S. Ruesga (coord.). Hacia un Sistema Nacional Público de Salud en México (p.19). Unam.

Méndez, J. (2017). Afiliación, uso y gasto en salud: ENIGH 2016. http://ciep. $\mathrm{mx} /$ afiliacion-uso-y-gasto-en-salud-enigh2016

(2019). La contracción del gasto per cápita en salud: 2010-2020. https://ciep.mx/la-contraccion-del-gasto-per-capita-en-salud-2010-2020

Mills, A. (2014). Health care systems in low- and middle-income countries. The New England Journal of Medicine, 370(6). https://doi.org/10.1056/ NEJMra1110897 
Mora-Rivera, J., Llamas, I. y García, M. (2018). Pobreza y hogares receptores de remesas a veinte años del tLCan: México 2010, 2012 y 2014. Economía Teoría y Práctica, (4). https://doi.org/10.24275/ETYPUAM/NE/ E042018/Mora

Murillo, B. y Almonte, L. (2020). Gasto público en salud y su composición, el caso de México. Economía Actual, 13(3). https://bit.ly/3ISUTAp

Organización de las Naciones Unidas (onU) (2019). Marco de indicadores mundiales para los Objetivos de Desarrollo Sostenible y metas de la Agenda 2030 para el Desarrollo Sostenible. https://bit.ly/3fhQ8hg

Organización Mundial de la Salud (oms) (2017). Seguimiento de la cobertura sanitaria universal: Informe de monitoreo global 2017. https://apps.who. int/iris/handle/10665/310924.

Pavón-León, P., Reyes-Morales, H., Martínez, A., Méndez-Maín, S., Gogeascoechea-Trejo, M. y Blázquez-Morales, M. (2017). Gasto de bolsillo en adultos mayores afiliados a un seguro público de salud en México. Gaceta Sanitaria, 31(4). http://dx.doi.org/10.1016/j.gaceta.2016.12.015

Pérez, R., Doubova, S., Wirtz, V., Dreser, A., Serván-Mori, E. y Hernández, M. (2013). Consultorios médicos en farmacias privadas: efectos inesperados en el uso de servicios de salud y el acceso a medicamentos. https://bit. ly/2UIuc5E

Pérez-Cuevas, R. y Doubova, S. (2019). La experiencia con la atención primaria de salud en México. En F. Guanais, F. Regalia, R. Pérez-Cuevas y M. Anaya (eds.). Desde el paciente. Experiencias de la atención primaria de salud en América Latina y el Caribe (pp. 125-148). Banco Interamericano de Desarrollo: División de Protección Social y Salud. http://dx.doi. org/10.18235/0001255

Salinas-Escudero, G., Carrillo-Vega, M., Pérez-Zepeda, M. y García-Peña, C. (2019). Gasto de bolsillo en salud durante el último ańo de vida de adultos mayores mexicanos: análisis del enasem. Salud Pública, 61(4). https://doi. org/10.21149/10146

Secretaría de Salud (ssa) (2019a). Informe de Resultados del Sistema de Protección Social en Salud Enero-Junio 2019. https://bit.ly/3pNllxD

(2019b). sicuentas Cierre Estadístico del Subsistema de Cuentas en Salud a Nivel Federal y Estatal 2017. https://bit.ly/3fl0PzM

Séne, L. y Cissé, M. (2015). Catastrophic out-of-pocket payments for health and poverty nexus: Evidence from Senegal. International Journal of Health Economics and Management, 15(3). http://dx.doi.org/10.1007/s10754015-9170-4 
Sistema de Cuentas en Salud a Nivel Federal y Estatal (sicuentas) (2019). Gasto en Salud en el Sistema Nacional de Salud. https://bit.ly/333IMJy

Soto-Estrada, G., Moreno-Altamirano, L. y Pahua, D. (2016). Panorama epidemiológico de México, principales causas de morbilidad y mortalidad. Revista de la Facultad de Medicina de la UNAM, 59(6). https://bit.ly/38Xie0i

Van Minh, H., Phuong, N., Saksena, P., James, C. y Xu, K. (2013). Financial burden of household out-of pocket health expenditure in Viet Nam: Findings from the National Living Standard Survey 2002-2010. Social Science \& Medicine, (96). https://doi.org/10.1016/j.socscimed.2012.11.028

Wirtz, V., Santa-Ana-Tellez, Y., Serván-Mori, E. y Ávila-Burgos, L. (2012). Heterogeneous effects of health insurance on out-of-pocket expenditure on medicines in Mexico. Value in Health, 15(5). https://doi.org/10.1016/j. jval.2012.01.006

Wirtz, V., Serván-Mori, E., Dreser, A., Heredia, I. y Ávila-Burgos, L. (2013). Surtimiento y gasto en el acceso a medicamentos en instituciones públicas: asignaturas pendientes. https://bit.ly/391a8Ur

Xu, K., Evans, D., Kawabata, K., Zeramdini, R., Klavus, J. y Murray, C. (2003). Household catastrophic health expenditure: A multicountry analysis. The Lancet, 362(9378). https://doi.org/10.1016/S0140-6736(03)13861-5

Xu, K., Evans, D., Carrin, G., Aguilar-Rivera, A., Musgrove, P. y Evans, T. (2007). Protecting households from catastrophic health spending. Health Affairs, 26(4). https://doi.org/10.1377/hlthaff.26.4.972 
